Acta Crystallographica Section D

Biological

Crystallography

ISSN 0907-4449

Yanhui Xu, ${ }^{a, b}$ Nan Su, ${ }^{a, b}$ Lan Qin, $^{\text {a,b }}$ Zhihong Bai, ${ }^{\text {a,b }}$ George F. Gao $^{c}$ and Zihe Rao ${ }^{a, b_{*}}$

a Laboratory of Structural Biology, Tsinghua University, Beijing 100084, People's Republic of China, ${ }^{\mathbf{b}}$ National Laboratory of Biomacromolecules, Institute of Biophysics, Beijing 100101, People's Republic of China, and 'Institute of Microbiology, Chinese Academy of Sciences, Beijing 100080, People's Republic of China

Correspondence e-mail: raozh@xtal.tsinghua.edu.cn

\section{Crystallization and preliminary crystallographic analysis of the heptad-repeat complex of SARS coronavirus spike protein}

The aetiological agent of an emergent outbreak of atypical

Received 2 September 2004 pneumonia, severe acute respiratory syndrome (SARS), is a Accepted 25 October 2004 positive-stranded RNA virus (SARS-CoV) belonging to the Coronaviridae family with a genome that differs substantially from those of other known coronaviruses. Highly conserved heptad-repeat (HR1 and HR2) regions in class I viral fusion proteins, including spike protein from SARS coronavirus, interact with each other to form a six-helix bundle, which is called a fusion core. The crystal structure of the fusion core is expected to greatly facilitate drug design. Crystals of the fusion core of SARS-CoV spike protein have been grown at $291 \mathrm{~K}$ using PEG 4000 as precipitant. The diffraction pattern of the crystal extends to $2.8 \AA$ resolution at $100 \mathrm{~K}$ in-house. The crystals have unit-cell parameters $a=121.2, b=66.3, c=70.0 \AA$, $\alpha=\gamma=90$, $\beta=107.4^{\circ}$ and belong to space group $C 2$. Assuming the presence of six molecules per asymmetric unit, the solvent content is estimated to be about $28 \%$.

\section{Introduction}

An emergent outbreak of atypical pneumonia, referred to as severe acute respiratory syndrome (SARS) and first identified in Guangdong Province, China, spread to several countries in the spring of 2003 (Marra et al., 2003). The National Microbiology Laboratory in Canada obtained the Tor2 isolate from a patient in Toronto and succeeded in growing a coronavirus-like agent in African green monkey kidney (Vero E6) cells (Marra et al., 2003). This coronavirus has been named publicly by the World Health Organization as 'SARS coronavirus' (Marra et al., 2003). This coronavirus has been identified as the distinct pathological entity responsible for severe acute respiratory syndrome (SARS), which infected more than 8000 people and killed 774 worldwide in 2003.

The coronaviruses are single-stranded positive-sense RNA viruses and are members of a family of enveloped viruses that include a large number of viruses that infect different animal species (Siddell et al., 1983). The predominant diseases associated with these viruses are respiratory and enteric infections, although hepatic and neurological diseases also occur (Siddell, 1995). Although morphologically a coronavirus, the SARS coronavirus (SARS$\mathrm{CoV}$ ) is not closely related to any of the three known classes of coronavirus and has been proposed to define a fourth class of coronavirus (group 4) (Rota et al., 2003).

The surface glycoproteins of enveloped viruses are essential for viral entry and repli- cation. These envelope proteins mediate both the initial virion attachment to the cell and the subsequent fusion of viral and cellular membranes, resulting in the release of the viral contents into the host cell (Eckert \& Kim, $2001 b$ ). As with other enveloped RNA viruses including the coronaviruses, genome sequencing reveals that SARS-CoV envelope spike (S) protein contains highly conserved heptadrepeat regions (HR1 and HR2), which have been shown to be important in virus membrane fusion. Such heptad-repeat regions have been successfully used as targets for virus entry/ fusion inhibitors in a number of viruses (Chan \& Kim, 1998; Eckert \& Kim, 2001b). It is generally believed that the envelope protein undergoes a series of conformational changes during the virus-fusion process. The HR1 and HR2 regions are believed to be important modules/domains in this process and show different conformations in different fusion states (Eckert \& Kim, 2001b). In the current model, there are at least three conformational states of the envelope fusion protein: the prefusion native state, the pre-hairpin intermediate state and the post-fusion hairpin state (Chan et al., 1997; Eckert \& Kim, 2001b). During these state transitions, HR1 and HR2 are exposed in an intermediate conformational state but bind to each other to form a coiledcoil structure in the post-fusion state. Therefore, the in vitro introduced HR peptides compete with their endogenous HR counterparts in the intermediate state, preventing the transition into the formation of HR1/HR2 coiled-coil bundle in the post-fusion state. 
Some HR peptides or analogues have been shown to be potent inhibitors of virus fusion (Eckert \& Kim, 2001a,b; Root et al., 2001).

Membrane fusion is the key step in enveloped virus infection. The coiled-coil bundle conformation is believed to be important for bringing two lipid membranes (cellular and viral) into proximity with each other, thus enabling the membrane fusion for virus entry. The HR1/HR2 coiled-coil bundle is also called the fusion core of the virus fusion protein (Eckert \& Kim, 2001b). Several crystal structures of fusion cores have been determined to date, including HIV gp41, HRSV F, influenza virus HA, Ebola virus GP and MHV (Bullough et al., 1994; Chan et al., 1997; Malashkevich et al., 1999; Xu, Liu et al., 2004; Zhao et al., 2000). We have solved the crystal structure of the fusion core of MHV spike protein and proposed a molecular mechanism for coronavirus-mediated viral fusion (Xu, Liu et al., 2004). However, the detailed structure of the SARS-CoV spike protein fusion core is still unknown.

Our previous studies have shown that the complex of HR1 and HR2 from the SARSCoV spike protein also forms a typical stable trimeric $\alpha$-helical bundle (Xu, Zhu et al., 2004). Here, we report the purification, crystallization and preliminary crystallographic studies of the fusion core of the SARS-CoV spike protein.

\section{Materials and methods}

\subsection{Expression and purification of SARS} fusion core (2-Helix) proteins

The SARS spike gene was cloned from SARS coronavirus GZ02 clone 1 (Gene Bank No. AY390556). The HR1 region used was derived from residues 900-948 and HR2 from residues 1145-1184. The SARS 2-Helix construct was made by linking the HR1 region and HR2 region with a 22 amino-acid

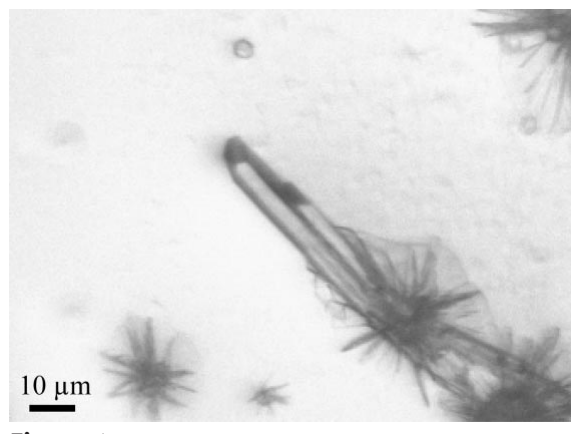

Figure 1

Typical crystals of the SARS 2-Helix protein grown by the hanging-drop method in $0.1 M$ sodium acetate $\mathrm{pH} \quad 2.5,13 \%(v / v)$ PEG 4000, $0.02 M$ spermidine. $4 \mathrm{HCl}$. linker (LVPRGSGGSGGSGGLEVLFQGP, single-letter amino-acid codes). The linker includes a thrombin-recognition site, a prescission recognition site and a typical flexible linker (GGSGGSGG). The constructs were cloned into the NdeI and XhoI sites (introduced by synthetic PCR primers) of the $\mathrm{pET} 22 \mathrm{~b}$ expression vector (Pharmacia). The construction was verified by sequencing, the expected plasmid was transformed into Escherichia coli strain BL21(DE3) competent cells and the transformants were selected on LB agar plates containing $100 \mu \mathrm{g} \mathrm{ml}^{-1}$ ampicillin. The cells were cultured at $310 \mathrm{~K}$ in $2 \times \mathrm{YT}$ medium containing $100 \mu \mathrm{g} \mathrm{ml}^{-1}$ ampicillin. When the culture density $\left(\mathrm{OD}_{600}\right)$ reached 0.8 , the culture was induced with $0.2 \mathrm{~m} M$ IPTG and grown for an additional $10 \mathrm{~h}$ at $289 \mathrm{~K}$ before the cells were harvested.

The bacterial cell pellet was resuspended in $1 \times$ PBS $(140 \mathrm{~m} M \mathrm{NaCl}, 2.7 \mathrm{mM} \mathrm{KCl}$, $\left.10 \mathrm{~m} M \mathrm{Na}_{2} \mathrm{HPO}_{4}, 1.8 \mathrm{~m} M \quad \mathrm{KH}_{2} \mathrm{PO}_{4}\right)$ and homogenized by sonication. The lysate was centrifuged at $18000 \mathrm{~g}$ for $20 \mathrm{~min}$ at $277 \mathrm{~K}$, the supernatant was loaded onto an $\mathrm{Ni}^{2+}$ NTA column (Qiagen), the contaminated unbound protein was washed off with $50 \mathrm{ml}$ washing buffer $(1 \times \mathrm{PBS}, 60 \mathrm{mM}$ imidazole $)$ and the target protein was eluted with elution buffer ( $1 \times$ PBS, $500 \mathrm{~m} M$ imidazole). The sample from affinity chromatography was concentrated to $0.5 \mathrm{ml}$ and subsequently loaded onto a Superdex 75 column HR10/30 (Pharmacia Biotech) column with an ÄKTA Purifier System (Pharmacia Biotech) in buffer containing $25 \mathrm{~m} M$ Tris- $\mathrm{HCl} \mathrm{pH} 8.0$, $150 \mathrm{~m} M \mathrm{NaCl}$. Protein purity was determined by $10 \%$ tricine SDS-PAGE.

\subsection{Crystallization}

The purified SARS 2-Helix protein was dialyzed against crystallization buffer (10 $\mathrm{m} M$ Tris- $\mathrm{HCl} \mathrm{pH} 8.0,10 \mathrm{~m} M \mathrm{NaCl}$ ) and concentrated to $10-15 \mathrm{mg} \mathrm{ml}^{-1}$. Crystallization was performed by the hanging-drop vapour-diffusion method at $291 \mathrm{~K} .1 \mu \mathrm{l}$ protein solution was mixed with $1 \mu \mathrm{l}$ reservoir solution and the mixture was equilibrated against $200 \mu \mathrm{l}$ reservoir solution at 291 K. Initial crystallization conditions were screened using Crystal Screen reagent kits (Hampton Research) and a PEG 4000 Screening kit prepared in-house. The protein could be crystallized under several conditions containing PEG 4000. Conditions yielding small crystals were further optimized by variation of precipitant, protein concentration, buffer $\mathrm{pH}$ and additives. Good-quality well diffracting crystals could be obtained in $0.1 M$ sodium acetate $\mathrm{pH} 2.5$,
Table 1

Data-collection and processing statistics for SARS 2-Helix.

\begin{tabular}{ll}
\hline Space group & $C 2$ \\
Unit-cell parameters $\left(\AA{ }^{\circ}\right)$ & $a=121.2, b=66.3$, \\
& $c=70.0, \beta=107.4$ \\
Wavelength $(\AA)$ & 1.5418 \\
Resolution limit $(\AA)$ & 2.8 \\
Observed reflections & 27312 \\
Unique reflections & 11974 \\
Completeness $(\%)$ & $91.4(80.8)$ \\
$I / \sigma(I)$ & $5.7(1.2)$ \\
$R_{\text {merge }}(\%)$ & $13.9(51.1)$
\end{tabular}

$\dagger R_{\text {merge }}=\sum_{h} \sum_{i}\left|I_{i h}-\left\langle I_{h}\right\rangle\right| / \sum_{h} \sum_{i}\left\langle I_{h}\right\rangle$, where $\left\langle I_{h}\right\rangle$ is the mean of the observations $I_{i h}$ of reflection $h$.

$10-15 \%(v / v)$ PEG 4000, $0.02 M$ spermidine. $4 \mathrm{HCl}$. The crystals appeared in two weeks.

\subsection{Data collection and processing}

The SARS 2-Helix crystal was mounted in a nylon loop and flash-cooled in a cold nitrogen-gas stream at $100 \mathrm{~K}$ using an Oxford Cryosystems coldstream with $0.1 \mathrm{M}$

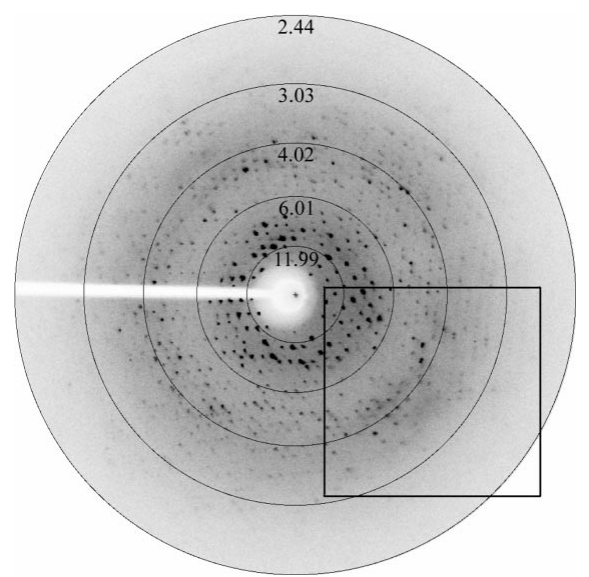

(a)

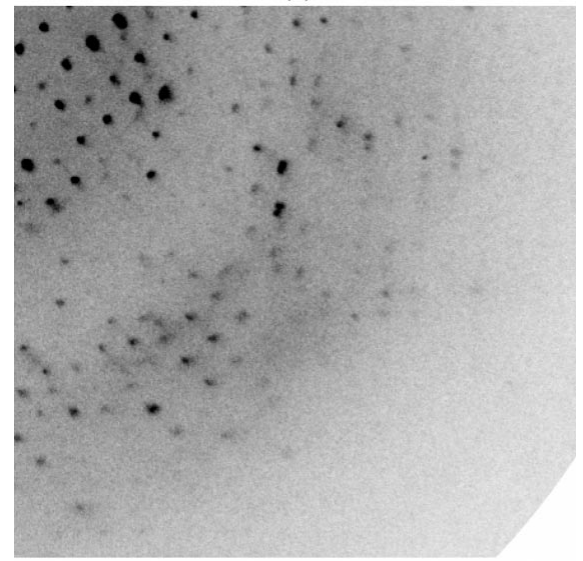

(b)

Figure 2

(a) A typical X-ray diffraction pattern from a crystal of SARS 2-Helix. The diffraction image was collected on a MAR 345 image-plate detector. The oscillation range is $1^{\circ}$. (b) An enlarged image of the area indicated in Fig. 2(a). 
sodium acetate $\mathrm{pH} 2.5,25 \%$ PEG 4000 as cryoprotectant. Data collection was performed in-house on a Rigaku RU2000 rotating copper-anode $\mathrm{X}$-ray generator operated at $48 \mathrm{kV}$ and $98 \mathrm{~mA}(\mathrm{Cu} \mathrm{K \alpha}$; $\lambda=1.5418 \AA$ ) with a MAR 345 image-plate detector. Data were indexed, integrated and scaled using DENZO and SCALEPACK (Otwinowski \& Minor, 1997).

\section{Results and discussion}

The SARS-CoV 2-Helix protein construct could be crystallized easily under several conditions, while good-quality crystals could only be obtained using $0.1 \mathrm{M}$ sodium acetate $\mathrm{pH} 2.5,13 \%(v / v)$ PEG 4000, $0.02 M$ spermidine. $4 \mathrm{HCl}$ (Fig. 1). Diffraction data were collected to $2.8 \AA$ from a single crystal. The crystals belong to space group $C 2$, with unitcell parameters $a=121.2, b=66.3, c=70.0 \AA$, $\alpha=\gamma=90, \beta=107.4^{\circ}$. Assuming the presence of six molecules (two stable fusioncore complexes) in the asymmetric unit, the Matthews number $\left(V_{\mathrm{M}}\right)$ is about $1.7 \AA^{3} \mathrm{Da}^{-1}$ and the solvent content is calculated to be about $28 \%$. Selected data statistics are shown in Table 1.

X-ray diffraction data of 2-Helix of SARS-CoV were collected from a single crystal using a Rigaku RU2000 rotating copper-anode X-ray generator and a MAR 345 image-plate detector (Figs. $2 a$ and $2 b$ ). The crystal-to-detector distance was $120 \mathrm{~mm}$, the exposure time was $600 \mathrm{~s}$, the oscillation angle was $1^{\circ}$ and the wavelength was $1.5418 \AA$.

The collected data were indexed and reduced with DENZO and SCALEPACK (Otwinowski \& Minor, 1997). The crystal structure of the SARS 2-Helix has been determined by molecular replacement with the MHV 2-Helix structure (PDB code $1 w d f)$ as a search model. Clearly, the crystal structure of the fusion core of SARS-CoV spike protein will provide a detailed picture of the viral fusion-core structure and the viral fusion mechanisms mediated by the SARS coronavirus spike protein. This will open a new avenue towards the structurebased fusion inhibitor design of peptides or peptide analogues, e.g. small molecules, for this emerging infectious disease.

This work was supported by Project 973 and 863 of the Ministry of Science and Technology of China [grant Nos. 200BA711A12, G199075600, GZ236(202/9) and 2003CB514103].

\section{References}

Bullough, P. A., Hughson, F. M., Skehel, J. J. \& Wiley, D. C. (1994). Nature (London), 371, 3743.

Chan, D. C., Fass, D., Berger, J. M. \& Kim, P. S. (1997). Cell, 89, 263-273.

Chan, D. C. \& Kim, P. S. (1998). Cell, 93, 681684.

Eckert, D. M. \& Kim, P. S. (2001a). Proc. Natl Acad. Sci. USA, 98, 11187-11192.

Eckert, D. M. \& Kim, P. S. (2001b). Annu. Rev. Biochem. 70, 777-810.

Malashkevich, V. N., Schneider, B. J., McNally, M. L., Milhollen, M. A., Pang, J. X. \& Kim, P. S. (1999). Proc. Natl Acad. Sci. USA, 96, 26622667.

Marra, M. A. et al. (2003). Science, 300,1399-1404.

Otwinowski, Z. \& Minor, W. (1997). Methods Enzymol. 276, 307-326.

Root, M. J., Kay, M. S. \& Kim, P. S. (2001). Science, 291, 884-888.

Rota, P. A. et al. (2003). Science, 300, 1394-1399.

Siddell, S. G. (1995). The Coronaviridae: an Introduction, pp. 1-10. New York: Plenum.

Siddell, S., Wege, H. \& Ter Meulen, V. (1983). J. Gen. Virol. 64, 761-776.

Xu, Y., Liu, Y., Lou, Z., Qin, L., Li, X., Bai, Z., Pang, H., Tien, P., Gao, G. F. \& Rao, Z. (2004). J. Biol. Chem. 279, 30514-50322.

Xu, Y., Zhu, J., Liu, Y., Lou, Z., Yuan, F., Liu, Y., Cole, D. K., Ni, L., Su, N., Qin, L., Li, X., Bai, Z., Bell, J. I., Pang, H., Tien, P., Rao, Z. \& Gao, G. F. (2004). In the press.

Zhao, X., Singh, M., Malashkevich, V. N. \& Kim, P. S. (2000). Proc. Natl Acad. Sci. USA, 97, 14172-14177. 